\title{
Allocation strategy for an ambulance base under traffic congestion
}

\author{
Suriyaphong Nilsang ${ }^{1}$, Chumpol Yuangyai ${ }^{1 *}$, Sirisuda Buatongkue $^{2}$, and Chen-Yang Cheng ${ }^{3}$ \\ ${ }^{1}$ Department of Industrial Engineering, Faculty of Engineering, King Mongkut's Institute of Technology Ladkrabang, Bangkok, Thailand \\ ${ }^{2}$ Department of Information technology, Faculty of Science and Arts, Burapha University, Chanthaburi, Thailand \\ ${ }^{3}$ Department of Industrial Engineering \& Management National Taipei University of Technology (Taipei Tech), Taipei, Taiwan
}

\begin{abstract}
One of crucial issues for emergency medical service (EMS) is to reduce response time. However, in metropolis city, a traffic congestion is an obstacle for an ambulance to responsively reach at the scene, then patient mortality and disability rates increase. Traffic congestion is considered as a complex spatialtemporal situation. It is often triggered by repeating factors, such as car lane capacity, weather, and unexpected events. Therefore, a real-time traffic condition is required to effectively determine the location of an ambulance. The current ambulance base allocation strategy model considers only demand point, resulting inability to handle high traffic congestion. This paper proposed a covering model based on traffic congestion (using Google map API) to allocate ambulance bases that covering all demand point, while minimizing the number of the ambulance. In addition, our model was applied to the case study of Bangkok EMS.
\end{abstract}

\section{Introduction}

One of crucial issues for emergency medical service (EMS) in metropolis city is traffic congestion. Especially, the ambulance services are highly affected. Their response time increases leading to the causes of death and disabilities of patients in crisis, which impose severe socio-economic costs across the world. [1]. The main objective of emergency ambulance service is to reach the scene within the effective target time for treatment. Typically, EMS responds to $90 \%$ of emergency calls within $8 \mathrm{~min}$ [2].

For example, the traffic condition for Bangkok is considered as the second-most congested city in the world, it has $61 \%$ congestion level (overall travel time when compared uncongested), which is 64 minutes of extra travel time (TomTom Traffic 2016). Extra travel time of traffic congestion affect to ambulance and survival rate of patients in the scene [3], therefore allocation strategy of ambulance has been affected by this traffic congestion. However, it is impossible to place an ambulance all possible bases. EMS managers and administrators have difficulties of locating a limited number of ambulances to cover all accidents or demands points.

Allocation strategy for ambulance have been studied for over 40 years, mostly called "covering model". Researchers have discussed two major models for the allocation of ambulance bases, such as a location set covering problem (LSCP) [4] and a maximal covering location problem (MCLP) [5], The latter is useful in solving strategic problems, in which the MCLP model lacks the flexibility required to solve operational problems because real-time demands vary spatially, temporally. For general covering model consider only a demand point covered and a number of ambulances to reduce response time. In practice, traffic congestion, patient conditions, public events, and geographical location of calls/ bases are also important. Specifically, this paper considers a covering model based on real time traffic congestion using information form Google Map API.

This paper is organized as follows: detailed description of traffic information are presented in Section 2. The overview of integrated model with traffic condition is discussed in Section 3. The Bangkok case is discussed in Section 4. The results, conclusion, and future work are in Section 5.

\section{Traffic Congestion Problem}

Traffic congestion is considered as a complex spatial-temporal situation. It is often triggered by repeating factors, such as car lane capacity, weather, and unexpected events (e.g. accidents, lane closures, and public events) [6]. Understanding those factors that cause congestion has become a critical issue in traffic management.

The causes of traffic congestion can be measured by physical and psychological factors. Physical causes measure the traffic volume, speed, and street density [7]. In June 2005 Google officially released their Google Maps Application Programming Interface (API), which enables users to mix Google streamed base data with other spatially referenced material that used Floating Car Data (FCD) or Floating Phone Data (FPD) that using the Global Positioning System (GPS). These data can then

* Corresponding author: chumpol.yuangyai@gmail.com 
be served as another applications through the Google map interface.

Therefore, this study bring traffic information (traffic speed) from Google map API with ambulance deployment on allocation strategic (covering model) to determine ambulance location that considering environment of traffic. Moreover, we considering number of ambulance on each bases that reduce response time and satisfied traffic speed in this area.

\section{Our Proposed Model}

The main purpose of this study was to use traffic information from Google map for the redeployment of the ambulances. An overview of the system in this study is shown in Figure 1. When high the traffic congestion occurs, traffic information and images at the scene shared to social media such as Google map, which publishes traffic information. We used this information to analyze traffic speed in data per unit period by filtering based on spatial-term, and temporal-term using floating phone data (FPD) of Google traffic for calculate traffic speed each main road and evaluate traffic congestion; moreover, we updated the ambulance base locations in next period.

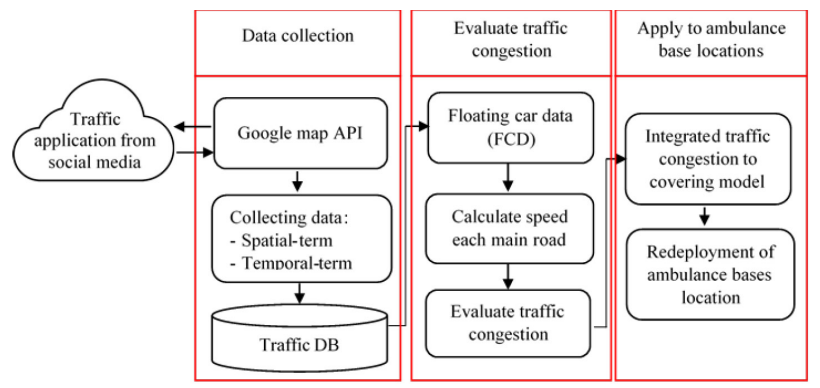

Fig. 1. Overview of integrated model of ambulance allocation with traffic condition from Google map.

\subsection{Data collection of traffic congestion from Google map}

The proposed framework of integrated traffic information for allocating ambulance bases is shown in Figure 1. First of all, we collected a traffic information database from Google map by using Google map API, that publish traffic information to study in this area information, such as main road, and level of traffic congestion (color on road) and average of car speed, that is shown in Figure 2. We then filtered spatial terms and temporal terms from the collected data in assigned area. In assigned area, there are more than one road and each line has a different road length, thus this study use average speed of car on this area to identity traffic condition. After got information, We compute the traffic speed on each area for allocate ambulance base by using the ratio of Traffic speed of road and a number of road in the area.

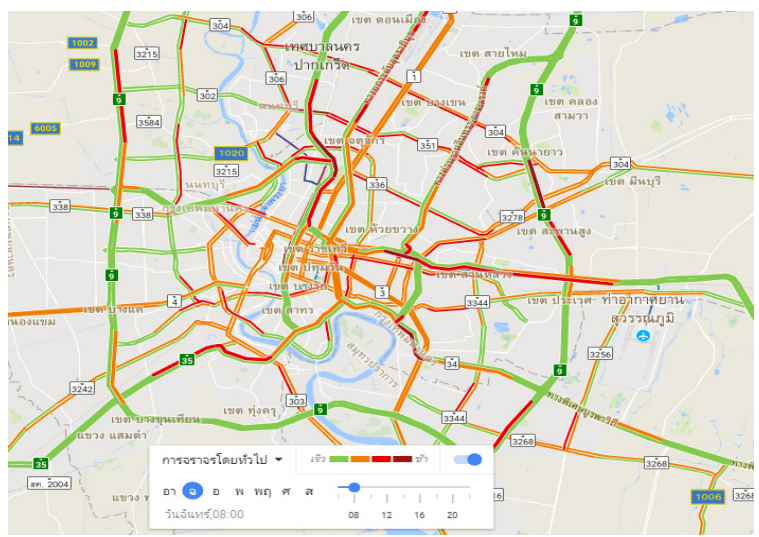

(a)

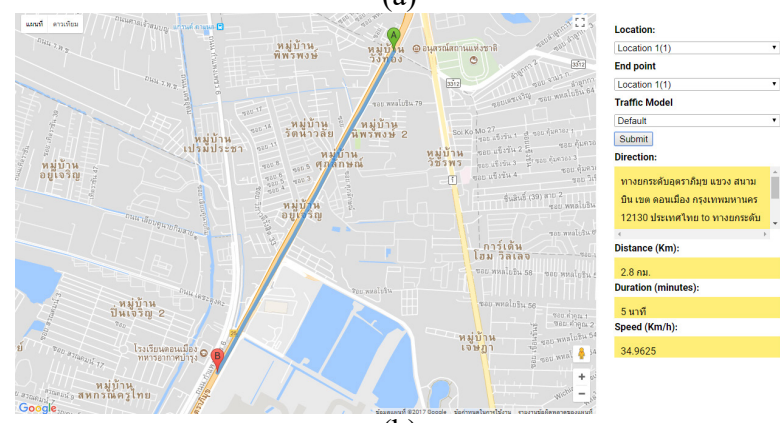

(b)

Fig. 2. (a) Google map that show traffic information, which indicate by color on road network. (b) Interface of google map API, which used for collect data of traffic information.

\subsection{Mathematical model with traffic condition}

We allocate both number of ambulances and base locations by using the covering model. In this study, we consider traffic congestion information into the model by adding a constraint to balance between average car speed and acceptable speed in a location. We named the proposed model as "Traffic Speed of Location Set Covering Problem for Traffic Speed" (LSCP-TS). Our model was under the following assumptions: traffic speed are deterministic, the base locations must be available in advance and all location can be ambulance base.

\subsubsection{Notation}

The model description is given as:

$$
\begin{array}{ll}
V & =\text { Set of demand points } \\
W & =\text { Set of potential base locations } \\
A_{i j} & =\text { Set of potential base locations that }
\end{array}
$$

\begin{tabular}{|c|c|c|}
\hline$i$ & $=$ & Index of demand points, $i \in V$ \\
\hline$j$ & $=$ & Index of potential base locations, $j \in W$ \\
\hline$P$ & $=$ & Total potential base location \\
\hline$C_{i}$ & $=$ & Average of speed in location $i$ \\
\hline$S_{i}$ & $=$ & Acceptable car speed in location $i$ \\
\hline$B_{j}$ & & $\begin{array}{l}\text { if base location is location - forced } \mathrm{j} \\
\text { otherwise }\end{array}$ \\
\hline$x_{j}$ & $=\left\{\begin{array}{l}1 \\
0\end{array}\right.$ & $\begin{array}{l}\text { if base location is selected at base } \mathrm{j} \\
\text { otherwise }\end{array}$ \\
\hline
\end{tabular}
cover demand point $i$ within the distance standard. 


$$
y_{i k}= \begin{cases}1 & \text { if demand point } \mathrm{i} \text { is covered } \text { at } \mathrm{k} \\ 0 & \text { otherwise }\end{cases}
$$

\subsubsection{Mathematical model}

This mathematical model is extended from modified covering model of Nilsang et al. [8] in location-forced constraint to determine impotent location to model. This can be formulated as follows:

$$
\begin{aligned}
& \text { Minimize } \quad \sum_{j \in W} x_{j} \\
& \text { Subject to } \quad \sum_{j \in W} A_{i j} x_{j} \geq \sum_{k=1}^{P} y_{i k} \quad i \in V \\
& \sum_{k=1}^{P} y_{i k} \geq 1 \quad i \in V \\
& \beta_{j} \leq x_{j} \quad j \in W \\
& \sum_{i=V} C_{i} \sum_{k=1}^{P} y_{i k} \geq S_{i} \theta \quad i \in V \\
& y_{i k} \in\{0,1\} \quad i \in V, k=1, \ldots, P \\
& x_{j} \text { Integer } \quad j \in W
\end{aligned}
$$

The objective of the model is to minimize number of ambulance base that cover all demand points while observing traffic congestion in all location. Traffic congestion occurred, ambulance vehicle may not be able to arrival to the scene if there is only one ambulance. Therefore, if the scene is covered by multiple bases and covered more than one ambulance, the scene will be taken care within target time. The objective function is given in (1). Constraint (2) and (3) ensures that ambulance base location cover demand point within the distance standard least than once. Sometime, allocation of ambulance base must assigned specific locations (e.g., public events) that shows in constraint (4). And our proposed constraint that consider traffic condition with average of traffic speed in this location must more than acceptable speed per one ambulance in constraint (5). Otherwise, that is variable constraints $x_{j}$ is integer and $y_{i k}$ is binary variable.

\subsection{Traffic information constraint into allocation strategy}

The integrated model, which is covering model with traffic condition and traffic data from Google map information can be used to determine ambulance base location in the future and reduce response time each periods of daily. Moreover, we added location-forced constraints that can be used when abnormalities (increasing accidents or public events) are observed on social media information. Both constraints traffic constraint and location-forced constraint are more useful on the strategic and operational levels.

For traffic speed constraint, we used Google Maps API for evaluate average traffic speed in real-time. Then we added traffic condition to our covering model (LSCPTS) to determine new ambulance base locations that use minimize ambulance base location and number of ambulance. To accept traffic speed that ambulance can arrive to the scene under target response time. For the new ambulance base, we set a new position of an ambulance base by using a geographic information system to set positions on a real map and we checked the covered demand points.

\section{A Case Study in Bangkok}

Bangkok is the capital and the most populous city in Thailand. Bangkok has a population of more than 14 million. Traffic congestion is major problem in Thailand, which has a tremendous impact on the economy and public health. In 2016 The INRIX Global Traffic Scorecard rated Bangkok the 12th most congested of all cities rated (1st most congested of Asia), considerably worse than 30th in 2015, with a traffic scorecard rating of 11, down from 20 in 2015 (INRIX 2017). Bangkok drivers spent an average 64.1 hours a year in traffic jams, according to the scorecard $23 \%$ of overall time and an average $33 \%$ of their time during peak hours. Moreover,

For historical traffic status in Bangkok area (google map 2017), it shows in Figure 3. We divided the area into 56 locations ( 6 kilometers by 6 kilometers) include 44 full of area and 12 some part of area and have 3 levels of traffic speed; (i) green area, average traffic speed more than $50 \mathrm{~km} / \mathrm{h}$, (ii) orange area, average traffic speed lower than $50 \mathrm{~km} / \mathrm{h}$ and more than $30 \mathrm{~km} / \mathrm{h}$, and finally (iii) rad area, average traffic speed lower than 30 $\mathrm{km} / \mathrm{h}$. that indicated most area in Bangkok have traffic speed lower than $50 \mathrm{~km} / \mathrm{h}$. At 8 minutes of response time, ambulance must travel to the scene by traffic speed 75 kilometers per hour.

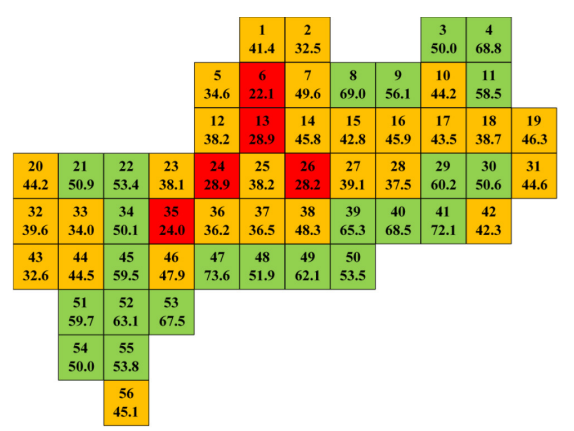

Fig. 3. Fifty-six areas of Bangkok and average traffic speed 3 level that show traffic problem in Bangkok.

\section{Results}

For results, we compare difference 3 periods, which difference traffic speed at each location. We collect traffic speed data from Google map API at 3 periods, such as daytime in weekend (assume all location traffic speed $80 \mathrm{Km} / \mathrm{h}$ ), peck hour in normally day and daytime in normally day to analyze traffic condition of each locations. Afterthought, we setting parameters follow that; potential ambulance base $(P)$ is 30 bases, traffic speed acceptance $\left(S_{i}\right)$ is $75 \mathrm{Km} / \mathrm{h}$ (Basically smooth) 
and location-forced $\left(B_{j}\right)$ is location No 27 for determine ambulance base locations by LSCP-TS model.

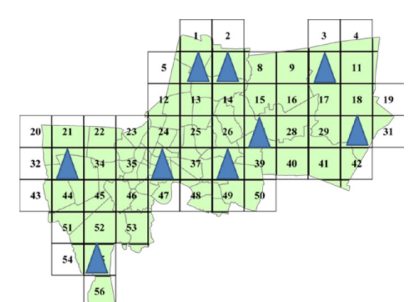

(a) Daytime in weekend

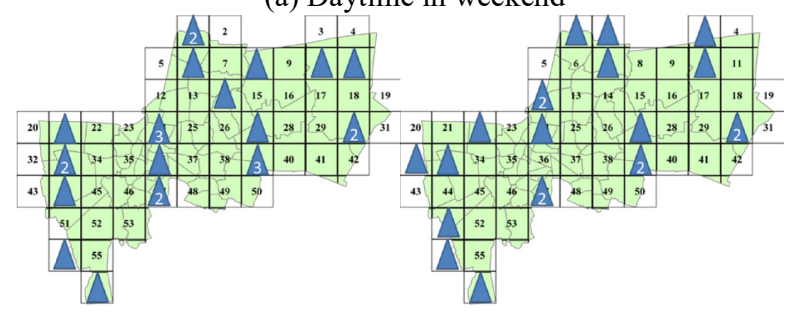

(b) Peck hour in normally day (c) Daytime in normally day

Fig. 4. Fifty-six areas of Bangkok and average traffic speed 3 types that show traffic problem in Bangkok.

After using the LSCP-TS model, the number of locations was 9 and 9 number of ambulances for daytime in weekend, which we used result as a base line to compare with another results. Two more results, daytime in weekday and peak hour in weekday, the number of locations is 17 bases, but difference location and number of ambulance (21 ambulances and 25 ambulances, respectively) to accept traffic speed, which show in Figure 4. It can be seen that LSCP-TS modal provide ambulance bases for daytime more than those of peak hour, because of a low traffic speed in peak hour so that model must add ambulance base to system. Although LSCP-TS model determine ambulance bases and ambulance vehicles a lot, but it can alleviate the effect of traffic congestion.

\section{Conclusions and Discussion}

In this study, we proposed an integrated framework of traffic condition, LSCP-TS constraints, to update ambulance base locations in response to traffic problem in urban. We explained the application of social media information for evaluate traffic condition, in the context of the EMS, and proposed an allocation model that does not require periodic relocation. We also validated the model by applying it to Bangkok EMS. Result showed that the locations of ambulance bases obtained that difference period from the proposed approach can cater to acceptable traffic speed and minimize the response times of ambulances under traffic conditions.

In future research, the relationship between uncertainty in risk management and the allocation of ambulance bases from social media information, will be considered. In general covering model for ambulance, we used only demand that not enough for ambulance allocation strategy. Therefore, we will focus on additional factors that related with ambulance management such as population density, disaster area, and public event etc.

\section{References}

1. Anand, J. and T.A. Flora, Emergency Traffic Management for Ambulance using Wireless Communication. IPASJ International Journal of Electronics \& Communication (IIJEC), 2014. 2(7): p. 43-52.

2. De Maio, V.J., et al., Optimal defibrillation response intervals for maximum out-of-hospital cardiac arrest survival rates. Annals of emergency medicine, 2003. 42(2): p. 242-250.

3. Karaman, M., A Genetic Algorithm for the Multi-Level Maximal Covering Ambulance Location Problem. Master of Science Thesis, 2008.

4. Toregas, C., et al., The location of emergency service facilities. Operations research, 1971. 19(6): p. 1363-1373.

5. Church, R. and C. ReVelle. The maximal covering location problem. in Papers of the Regional Science Association. 1974. Springer.

6. Kwon, J., M. Mauch, and P. Varaiya, Components of congestion: Delay from incidents, special events, lane closures, weather, potential ramp metering gain, and excess demand. Transportation Research Record: Journal of the Transportation Research Board, 2006(1959): p. 84-91.

7. Lu, J. and L. Cao. Congestion evaluation from traffic flow information based on fuzzy logic. in Intelligent Transportation Systems, 2003. Proceedings. 2003 IEEE. 2003. IEEE.

8. Nilsang, S., Yuangyai, C., Janjarassuk, U., and Cheng, C.Y. Locating an Ambulance Base by Using Social Media: A Case Study in Bangkok. Annals of Operations Research, (In-process of publish). 